\section{Programa de investigación y desarrollo orientado a problemas sociales y productivos}

Érica Hynes

Secretaria de Ciencia y Técnica.

Universidad Nacional del Litoral.

\section{Hugo Erbetta}

Secretario de Extensión Universitaria.

Universidad Nacional del Litoral.

\section{Introducción}

Las diversas formas de vinculación y articulación entre la universidad, como institución generadora de conocimiento científico y desarrollo tecnológico, y los problemas productivos y sociales que acontecen en la región configuran un debate actual. En los países desarrollados, sectores importantes de la sociedad demandan un papel más activo en la discusión de las agendas que determinan el curso de la ciencia y la tecnología. En América Latina, aunque no se logró aún tal nivel de avance en la discusión de estas agendas, se han desarrollado varios programas exitosos para promover investigaciones orientadas a problemas. ${ }^{1}$ En Argentina, la Universidad Nacional del Litoral fue pionera en la construcción de una herramienta de gestión que apuesta al financiamiento de líneas de investigación y desarrollo orientadas a problemas socialmente relevantes. Así, en 2007 se puso en marcha el Programa de Investigación y Desarrollo Orientado a Problemas Sociales y Productivos mediante el cual la Universidad profundiza el conocimiento y aporta soluciones sobre problemas que la comunidad y el desarrollo sostenible de la región demandan. El Programa incorpora un avance en la generación de conocimientos en el contexto de su aplicación, particularmente a través de la identificación de los problemas y de la participación de los beneficiarios de los proyectos. Busca articular los ámbitos académicos con los sociales durante todo el proceso de investigación, una práctica que interpela la metodología misma de producción del conocimiento. La apropiación social de los resultados, por otra parte, se incentiva mediante el acompañamiento en todas las instancias de preparación y ejecución del proyecto por los actores sociales que comparten la responsabilidad de identificar, por un lado, los problemas sociales y productivos y, por otro, las mejores maneras de aplicar los resultados de la investigación. En la actualidad transita su segunda convocatoria (2010), y ha finalizado recientemente los proyectos de la primera cohorte (2008).

\section{Propuesta}

Cuando la investigación asume características participativas y reconoce el valor del conocimiento de los sujetos sociales, más ricos y pertinentes son los resultados. Por lo tanto, se torna importante la inclusión de metodologías de investigación que permitan articular el saber cotidiano con el saber especializado, así como facilitar y propiciar el acceso de la sociedad a dichos resultados.

Como bien señala Abeledo (2003),

"los esquemas de gestión que promueven investigaciones orientadas a la solución de problemas tienen la virtud de crear las condiciones para un mejor aprovechamiento de los resultados y de generar mayores impactos socioeconómicos. Para promover investigaciones que tengan estas características se necesita un mayor esfuerzo de gestión y organización por parte de las agencias de política y gestión de ciencia y tecnología, pero los resultados demuestran, sin embargo, que este mayor esfuerzo organizativo conduce a una relación más fluida y provechosa entre las actividades de investigación y la sociedad que se beneficia por sus resultados".

En este sentido, el Programa surge como
1) Con relación a esto, son representativos ejemplos las Agendas de Investigación en Venezuela

—organizadas a partir del interés común por resolver un problema entre varias instituciones públicas y privadas-, las Mesas Sectoriales de Uruguay -constituidas como consorcios entre empresas que comparten problemas comunes e instituciones de investigación en algunos sectores productivosy el FONDEF de Chile - que cofinancia proyectos que incluyen desde su formulación la participación de los interesados en la solución de un problema particular-, entre otros. 
una estrategia de la Universidad para abordar cuestiones de su ámbito de pertenencia, que le permite profundizar el conocimiento y aportar soluciones sobre problemas $^{2}$ que la comunidad y el desarrollo sostenible de la región demandan.

Los temas ${ }^{3}$ que se identifican para cada convocatoria y los problemas derivados de los mismos, promueven la interacción disciplinaria como herramienta indispensable para afrontar de modo integrado las problemáticas a las que se pretenda dar respuesta. Esta integración, al tiempo que nuclea distintas disciplinas, favorece la labor conjunta de grupos de trabajo que, aunados en pos de objetivos comunes, se deben dar sus propias metodologías y estructuras de investigación (proyectos) acorde a las características del proceso a desarrollar. En síntesis, la formulación de esta nueva línea de proyectos, en el marco de un Programa de I+D de la UNL, en general, requiere:

- Definir los modos y procedimientos a través de los cuales se conformarán los grupos de trabajo que llevarán adelante los proyectos, y los mecanismos de asignación de los recursos.

2) Entendiendo el término problema como "aquel que surge de las exploraciones sobre el tema, o conjunto de conocimientos donde se inserta el interrogante específico al cual la investigación busca dar respuesta". Cf. Yúfera (1990).
- Establecer instancias de interacción en la formulación y en el desarrollo de los proyectos con los posibles destinatarios de los resultados de los mismos.

- Precisar los criterios de evaluación ex ante de los proyectos.

- Definir las instancias de seguimiento y de evaluación ex post de los proyectos.

Con relación a cada convocatoria particular del Programa, se procura:

- Identificar, seleccionar y priorizar los temas en torno a los cuales se desarrollarán las investigaciones.

- Identificar y seleccionar los problemas que serán objeto de estudio en el marco de los temas previamente seleccionados y priorizados.

\section{Temas de las convocatorias}

Para la identificación de los temas alrededor de los cuales posteriormente se enmarcan los problemas, se consideran criterios de carácter social y/o económico asociados con el bienestar general de la ciudadanía y vinculados al desarrollo local y regional. En 2010, los temas prioritarios retomaron aquellos de la primera convocatoria, ya que las problemáticas detectadas eran todavía de gran interés para el conjunto de la sociedad santafesina, y la estabilidad de los temas prioritarios se consideró deseable para aumentar impacto de la investigación. La experiencia brindada por la presentación y evaluación de ideas proyectos y proyectos, en 2008, y la ejecución del primer año de los mismos, constituyó un valioso aporte para la re-discusión de los temas. Participaron, además de las unidades académicas, las áreas centrales de Extensión, Planeamiento y Vinculación Tecnológica y Desarrollo Productivo, y consultores externos expertos en instrumentos de investigación-acción. Los temas preseleccionados se presentaron ante el Consejo Social de la UNL, que emitió opinión sobre los mismos, lo que se vio reflejado en nuevos ajustes a la formulación de las propuestas. Finalmente, se consultó al gobierno provincial, a través de la Secretaría de Estado de Ciencia, Tecnología e Innovación, y a los municipios de las ciudades donde la UNL tiene sede: Santa Fe, Esperanza, Gálvez y Reconquista. Sobre la base de
3) El tema puede ser definido, como refiere la cita anterior, como "conjunto de conocimientos donde se inserta el problema o interrogante específico" (Yúfera, 1990). 
una construcción colectiva que tuvo en cuenta las opiniones de todos los actores consultados, se llegó a la versión 2010 de los temas prioritarios.

Por otra parte, las líneas identificadas se confrontaron con las prioridades de otras instituciones de promoción y ejecución de ciencia y tecnología y de educación superior, con el objetivo principal de realizar esfuerzos convergentes y articular las políticas de promoción. Se consideraron: a) los temas prioritarios del Consejo Interuniversitario (CIN), seleccionados mediante la realización de talleres en todo el territorio nacional, en el marco del Programa Estratégico de Investigación y Desarrollo (PEID) del organismo y bajo la coordinación de su comisión de Ciencia, Técnica y Arte; b) las líneas prioritarias del Plan Estratégico Nacional de Ciencia, Tecnología e Innovación "Bicentenario" (2006-2010), que se reflejan en el diseño de los diversos instrumentos de la Agencia Nacional de Promoción Científica y Tecnológica, a través de sus fondos FONCyT, FONTAR y FONARSEC; c) los temas de interés provincial relevados por el Gobierno de la Provincia de Santa Fe, que son objeto de una convocatoria anual; d) los documentos surgidos de la construcción de consenso por parte de la comunidad internacional, tales como las publicaciones del Programa de las Naciones Unidas para el Desarrollo — Objetivos del Milenio-, y las agendas surgidas de reuniones de instituciones de educación superior (CMES, 2009; Guadalajara, 2010).

En 2011 dieron inicio 14 proyectos distribuidos en los temas:

1) Sustentabilidad, medio ambiente, gestión del riesgo Innovación en la sustentabilidad de procesos (tecnologías duras y/o de gestión). Evaluación, regulación, gestión y comunicación de riesgo de origen antrópico o natural. Sistemas energéticos sustentables.

\section{2) Alimentos y agua potable}

Producción de alimentos en el nuevo contexto rural. Accesibilidad y gestión del agua potable.

\section{3) Salud pública y vulnerabilidad social}

Patologías prevalentes en sectores socialmente vulnerables. Vulnerabilidad social y alimentación. Zoonosis.

4) Desarrollo territorial Trabajo y empleo. Desarrollo e inseguridad social. Expresiones de la economía social. Acompañamiento social a personas en situación de exclusión social. Prevención de la violencia y el delito. Educación para el trabajo. Asociaciones comunitarias e institucionales. Desarrollo de la competitividad de PyMEs. Territorio y urbanismo.
5) Escuela pública y sistema educativo Políticas públicas en educación. Escolarización de niños y jóvenes en sectores socialmente excluidos. Diseño y gestión del curriculum en la educación superior universitaria.

Es importante destacar que el Programa de Investigación Orientada de la UNL se constituyó en la primera experiencia en el seno de las universidades nacionales. Por sus características y alcances especiales implicó un aporte significativo al sistema universitario nacional. 


\section{Objetivos del Programa}

El Programa de Investigación y Desarrollo Orientado a Problemas Sociales y Productivos tiene como objetivos principales:

Vincular los espacios de producción de conocimientos con los de su uso y apropiación social.

- Articular los ámbitos académicos con los sociales durante el proceso de producción de conocimientos.

- Incentivar la investigación científica orientada a la atención de problemas sociales y productivos de la región.

- Promover la investigación conducente a resultados socialmente relevantes.

- Alentar la interdisciplinariedad en los proyectos.

\section{Bibliografía}

Abeledo, Carlos (2003). "Investigación orientada a la solución de problemas: relevancia y desafíos para países en desarrollo." Revista Interciencia, Vol. 28, n 10, octubre.

Yúfera, Eduardo (1990). "El proceso investigador", Capítulo 3. En Introducción a la investigación científica y tecnológica. Madrid: Alianza Universidad.

\begin{tabular}{lll} 
Convocatoria & $\begin{array}{l}\text { Cantidad } \\
\text { de proyectos } \\
\text { presentados }\end{array}$ & $\begin{array}{l}\text { Cantidad } \\
\text { de proyectos } \\
\text { financiados }\end{array}$ \\
\hline 2008 & 25 & 10 \\
\hline 2010 & 23 & 14 \\
\hline
\end{tabular}

